\title{
Verbal Aggression On Social Media: How, Why And Its AUTOMATIC IDENTIFICATION
}

\author{
RITESH KUMAR
}

\begin{abstract}
In recent times, verbal aggression and related phenomena of hate speech, abusive language, trolling, etc. have become a major problem over social media. In this paper, I present the results of a large-scale quantitative study of aggression based on a target-based typology in a manually-annotated multilingual dataset of over 20,000 Facebook comments and tweets each written in Hindi, English or code-mixed Hindi-English. Taking insights from this study, I develop 2 different classifiers for detecting aggression in Hindi, English and Hindi-English mixed Facebook and Twitter conversations. The classifiers are developed using an annotated corpus of approximately 9,000 Facebook comments and 5,000 tweets. Since a phenomenon like aggression is highly subjective, the study shows a comparatively modest inter-annotator agreement of 0.72 and an overall F1 score of 0.64 for both Facebook and Twitter. Consequently, I also carried out two user studies, where humans were asked to evaluate the annotations by the classifier, to test the actual 'acceptance' of the classifier's judgments. I discuss the results of this user study and give an analysis of the overall performance of the system.
\end{abstract}

KEYworDs: Verbal aggression, Facebook, Twitter, Hate speech, Politeness

\section{INTRODUCTION}

Over a period of last decade or so, with the emergence of an interactive web and especially popular social networking and social media platforms like Facebook and Twitter, there has been an exponential increase in the user-generated content being made available over the web. It has given the power to reach billions of people within a matter of seconds. This has resulted in not only benign exchange of ideas but has also led to a widespread dissemination of aggressive and potentially harmful content over the web. While most of the potentially harmful incidents like bullying or hate speech have predated the Internet, the reach and extent of Internet has given these incidents an unprecedented power and influence to affect the lives of billions of people. It has been reported that these incidents have not only created mental and psychological agony to the users of the web but has in fact forced people to deactivate their accounts and in rare instances also commit suicides. Thus the incidents of aggression and unratified verbal behaviouri has not remained just a minor nuisance but has acquired the form of a major criminal activity that affects a large number of people. So it is of utmost significance and importance that some preventive measures 
should to be undertaken to safeguard the interests of the people using the web as well as of the web such that it remains a viable medium of communication and connection, in general.

While the initial response to handling these aggressive instances of behaviour was to manually monitor and moderate the user-generated content, the amount and pace at which new data is being created over the web has rendered these manual methods of moderation and intervention almost completely impractical and ineffective. As such it has become imperative that such actions are recognised and dealt with using automatic or semi-automatic tools.

In this paper, I present a comparative analysis of types and extent of aggression over two of the most popular social media / networking platforms - Twitter and Facebook - based on a typology of aggression developed by us. Taking insights from this study, I discuss the development of a 3-class classifier for identifying aggression on social media. I also conducted a basic user survey to assess the acceptability of the classifier's judgments among the users.

\section{Verbal Aggression: Previous Literature and Typology}

Verbal aggression could be understood as any kind of linguistic behaviour which intends to damage the social identity of the target person and lower their status and prestige (Barron and Richardson 1994, cited in Culpeper 2011). It is any kind of behaviour that upsets the social equilibrium. In general, verbal aggression can be ratified as well as unratified and it is but obvious that we are mainly concerned with the unratified aggressive behaviour. In order to build an automatic aggressiondetection system, it is important that we have a good understanding of the structure of the phenomenon of aggression itself, especially the distinction between ratified and unratified behaviour such that the system flags only the most serious cases of aggression. Previous research in the field has been carried out to automatically recognise several related behaviours such as trolling (Cambria, et al., 2014; Kumar, Spezzano and Subrahmanian, 2013; Mojica, 2016; Mihaylov et al, 2015), cyberbullying (Kathick et al., 2012; Nitta et al., 2013; Dadvar, Trieschnigg and de Jong, 2014; Van Hee et al, 2015), flaming / insults (Sax, 2016; Nitin et al., 2012), abusive / offensive language (Chen et al, 2012; Nobata et al., 2016), hate speech (see Schmidt and Wiegand 2017 for a survey) and others. In addition to this, there have been some pragmatic studies on behaviour like trolling (Hardaker, 2010, 2013).

This huge interest in the field from different perspectives has created a huge conglomeration of terminologies as well as understandings of the phenomenon. This, on the one hand, provides us with a very rich and extensive insight into the phenomena but, on the other hand, has also created a theoretical gap in the understanding of interrelationship among these. While a large section of the research have focussed on any one of 
these phenomena and their computational processing, it seems there is a significant overlap among these phenomena in the way they are understood in these studies - and because of this underlying overlap, insights from different studies might prove useful for solving these seemingly different phenomena. All of these behaviours are considered undesirable, aggressive and detrimental for those on the receiving end. So, trolling is intended "to cause disruption and/or to trigger or exacerbate conflict for the purposes of their own amusement" (Hardaker, 2010). Cyberbullying is "humiliating and slandering behavior towards other people" (Nitta et al. 2013). Flaming intends "to offend someone through e-mail, posting, commenting or any statement using insults, swearing and hostile, intense language, trolling, etc." (Krol, 1992). Going purely by these understandings, the overlap among these phenomena are pretty obvious - as we try to classify actual data in one of these categories, the overlap becomes even more prominent. As such it might be possible to tackle all of these using similar methods, rather than building separate systems for each of these.

Verbal aggression could be divided into two basic types based on how it is expressed (these are called 'levels' of aggression in the aggression tagset) -

(c) Overt Aggression: Any speech / text in which aggression is overtly expressed - either through the use of specific kind of lexical items or lexical features which is considered aggressive and / or certain syntactic structures is overt aggression. Let us examine an example given below:

bjp wale jyada dhindhora pitate $h$ hindutva ka...aur hindu me hi equality nhi de pa rhe.isliye bjp kavirodh.baki states $k$ compare me bjp ruled state me ye jyada hota h.isliye $v$.

The BJP people brag about Hindutva more than others and they are not able to ensure equality among Hindus. That is why this opposition against BJP. And also because this happens more in the BJP-ruled states \footnote (BJP is considered pro-Hindu party in India. But in this comment, they are accused of highly discriminative attitude against Hindus (of upper caste). What I have translated as "brag" in English indicates a combination of hypocrisy and excessive advertisements in Hindi.

(d) Covert Aggression: Any text in which aggression is not overtly expressed is covert aggression. It is an indirect attack against the victim and is often packaged as (insincere) polite expressions (through the use of conventionalised polite structures), In general, lot of cases of satire, rhetorical questions, etc. may be classified as covert aggression. For example,

PhD kab poori karoge

When will you complete your $\mathrm{PhD}^{\mathrm{ii}}$ 
This classification is quite similar to the distinction in between 'Explicit' and 'Implicit' abuse proposed by Waseem, et al. (2017). In fact, the understanding of abuse in Waseem et al. (2017) is quite similar to our understanding of unratified aggression. However, they do not make a distinction between the ratified and unratified abuse because of the restrictions and the requirement that the use of the word 'abuse' puts on the researchers - abuse cannot be used in a positive sense, the way aggression can be.

Verbal aggression can be further divided into 4 different types based on the target of aggression (these are called 'discursive effects' ii in the aggression tagset) - Physical Threat, Sexual Threat / Aggression, Identity Threat / Aggression (further classified into 6 types viz, Gendered Aggression, Geographical Aggression, Political Aggression, Casteist Aggression, Communal Aggression, Racial Aggression) and Non-threatening Aggression.

This typology and an exhaustive, hierarchical tagset, based on this typology is discussed in detail in Kumar et al (2018) and is also included in the appendix of the paper. In the present study, we experiment with only 3 tags at the top level - Covertly Aggressive (CAG), Overtly Aggressive (OAG) and Non-aggressive (NAG)

\section{Data Collection and Annotation}

The data for the current corpus was crawled from the public Facebook Pages and Twitter. The data was mainly collected from the pages/issues that is expected to be discussed more among the Indians (and in Hindi).

For Facebook, more than 40 pages were recognised and crawled to collect the data. It included the pages of various kinds viz. news websites / organizations like NDTV, ABP News, web-based forums / portals like Firstpost, The Logical Indian, political Parties / groups like INC, BJP, students' organisations / groups like SFI, JNUSU, AISA, and other similar pages.

For Twitter, the data was collected using some of the popular hashtags around such contentious themes as beef ban, India vs. Pakistan cricket match, election results, opinions on movies, etc.

During collection, the data was not sampled on the basis of language and so it included data from English, Hindi as well as some other Indian languages. At a later stage, the data from languages other than Hindi and Hindi-English code-mixed were handled at a later stage.

A total of approximately 40,000 Facebook comments $(22,000$ in English and 18,000 in Hindi and Hindi-English code-mixed) and 20,000 tweets have been annotated using the top-level of the aggression tagset (i.e. levels of aggression) mentioned in the previous section. Moreover, 20,000 of these Facebook comments and all the tweets were also annotated for discursive 
effects, thereby, providing a rich dataset for studying aggression on social media as well as carrying out classification experiments.

A total of 8 annotators annotated the data in both the languages. All the annotators were research students in Linguistics and half of them have been working more generally in different sub-fields of computational linguistics. However, none of them were directly or indirectly involved with this research (except as annotators). The annotators were given a detailed and precise annotation guideline ${ }^{i v}$ and they were also able to discuss the contentious issues and solve their problems by talking to each other and also to the researchers.

Prior to the actual annotation task, an inter-annotator experiment was also conducted involving these 8 annotators as well as a large number of other annotators. The experiment was conducted using a crowdsourcing platform, Crowdflower, using approximately 1100 test instances. In these experiments, each instance was annotated by 3 annotators. A total of 77 annotators participated in the experiment. The platform allowed the annotators to remain anonymous to each other and so they did not have an opportunity to discuss their decision with each other. At the end of the experiments, the Krippendorff's Alpha was slightly above 0.72, which may seem quite low in comparison to the agreement in some other more objective tasksv. However, given the extremely subjective nature of metapragmatic judgments about aggression, the agreement could be considered rather respectable.

\section{LANGUAGE IDENTIFICATION OF THE COMMENTS}

In order to separate out comments from different languages, more than 81,000 comments were manually annotated at the comment-level for their language using a simple tagset with 4 tags - Hindi, English, Other and Hindi-English Code-mixed. The annotations were carried out by 9 annotators and were validated by 3 annotators. The dataset consisted of a total of over 37,000 each of Hindi and English, over 4,000 code-mixed data and over 2,500 comments from other languages. This dataset was used to develop a 4-class SVM-based classifier for automatic detection of language in comments. The classifier was trained using character 5 -grams combined with word trigrams using over 65,000 train instances and tested using over 16,000 train instances. The classifier gives the best overall F1 score of over $92 \%$ on the test set, with almost perfect scores for Hindi and English but a rather low score for mixed and other categories.

\section{AgGression on Social Media}

In order to understand the extent and nature of aggression on social media, we used the dataset of over 20,000 Facebook comments and tweets each with the aggression tagset at 2 levels - the level of aggression and the discursive effects. Using this tagged data, we tried to understand the 
following about the phenomena of aggression over both Facebook and Twitter:

- Is aggression different from abuse?

- Is aggression realised differently across different platforms?

- What is it that most of the people become aggressive about?

- How casteist, communal or racial are people on social media?

\section{AgGression vs Abuse}

One of the first observations about aggression is related to its relationship with abuse or abusive language. Abuses and aggression are often correlated but neither entails the other. However, both do occur together in a lot of cases and lot of times we are probably more concerned with (actual) abuses (and not the banter / teasing) than aggression itself. In cases of 'banter' and 'jocular mockery', abusive constructions are used for establishing inter-personal relationships and increasing solidarity. On the other hand, a lot of aggressive comments did not contain abuse (Figure 1). The data shows that only approx. 4\% of aggressive comments / tweets are abusive.

Figure 1: Proportion of abuse in aggressive Tweet and Facebook comments

Abuse in Aggression: Twitter

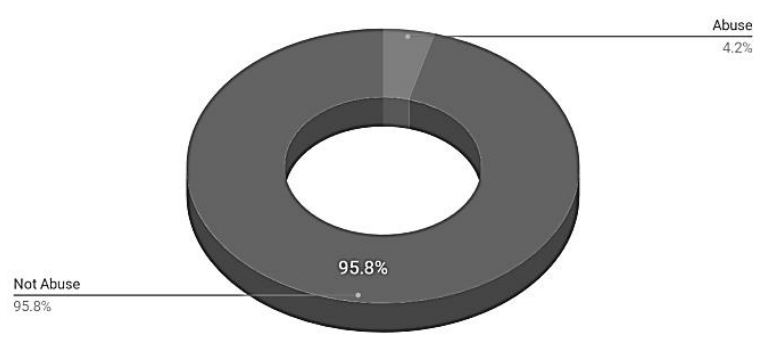

Abuse in Aggression: Facebook

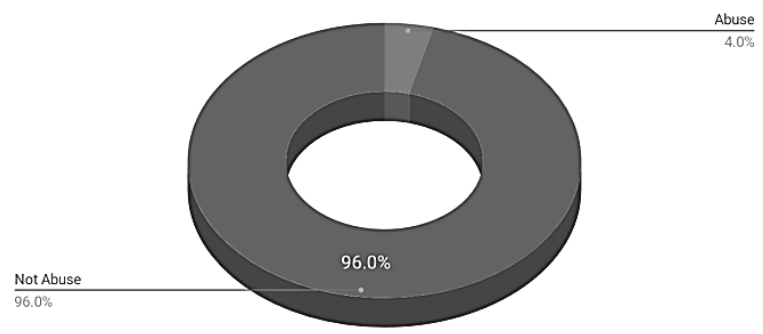

\section{AgGRession: TwitTer vs FACEBOOK}

Since we had annotated data from both of the two major social media platforms, we compared the extent and nature of aggression over the two platforms (Figure 2). The data shows a qualitative as well as quantitative difference in the use of aggression over the two platforms. While Facebook data had a slightly higher proportion of aggressive comments, there was a significant difference in the level of aggression across the two platforms Twitter contained a comparatively larger proportion of covertly aggressive comments while in Facebook both overtly and covertly aggressive comments were equally frequent. 
Figure 2: Proportion of aggressive comments on Twitter and Facebook

Tweets Proportion

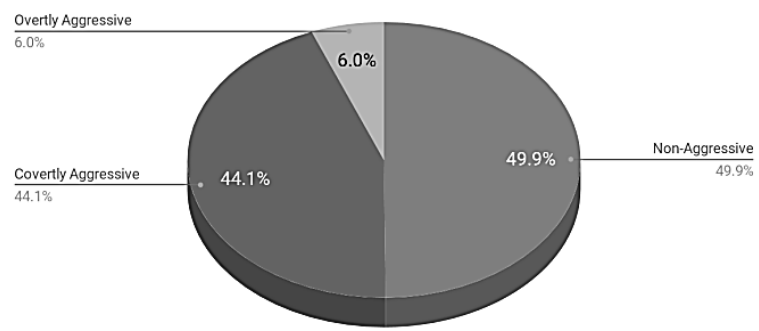

Facebook Comments Proportion

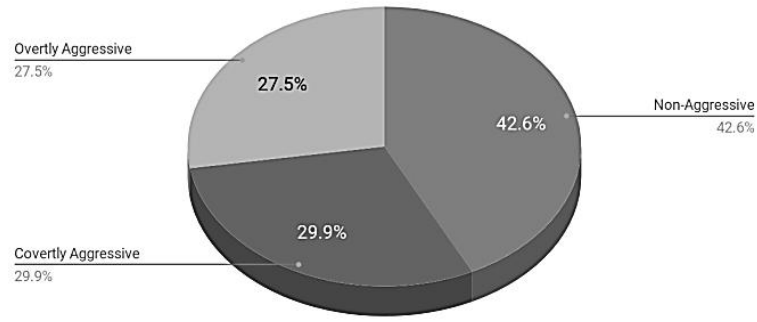

One of the obvious explanations for this distinction between Twitter and Facebook could be the length restrictions - it could be hypothesised that since Facebook does not impose any length restrictions, it allows users to be more aggressive. In order to test it, we took out a subset of Facebook dataset such that it contained 140 or less characters (same as Twitter) and compared the use of aggression (Figure 3). It turned out that length was not a significant factor as the proportion of covertly and overtly aggressive comments remained the same even after controlling for the length of Facebook comments. However, the proportion of non-aggressive comments because closer to that of Twitter. This distinction can also be because of the other factors like users' demography like age or socio-economic profile and needs to be further investigated.

Figure 3: Proportion of aggressive comments on Facebook (less than 140 characters)

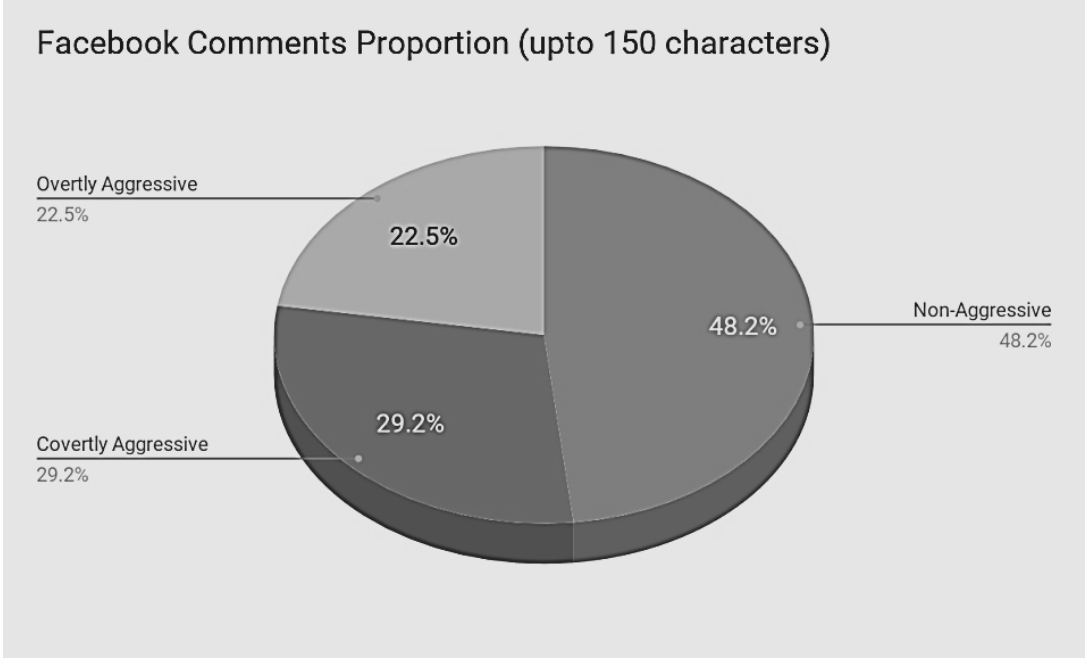

\section{WHAT MAKES PEOPLE AGGRESSIVE?}

The third important question relates to the reason or target of aggression on social media. Our study shows that most of the aggression is directed against identity or are non-threatening (Figure 4). However, even though physical threat and sexual threat are comparatively less, they can NEVER be negligible - even one such instance is a cause of extreme worry 
and warrants caution. Moreover, it is also to be noted that while identity threat and non-threatening aggression are almost equally common on both the platforms, the more serious threats like physical threat and sexual threat are much more prevalent on Facebook than on Twitter (Figure 4).

Figure 4: Different types of aggression and their proportion on the two platforms
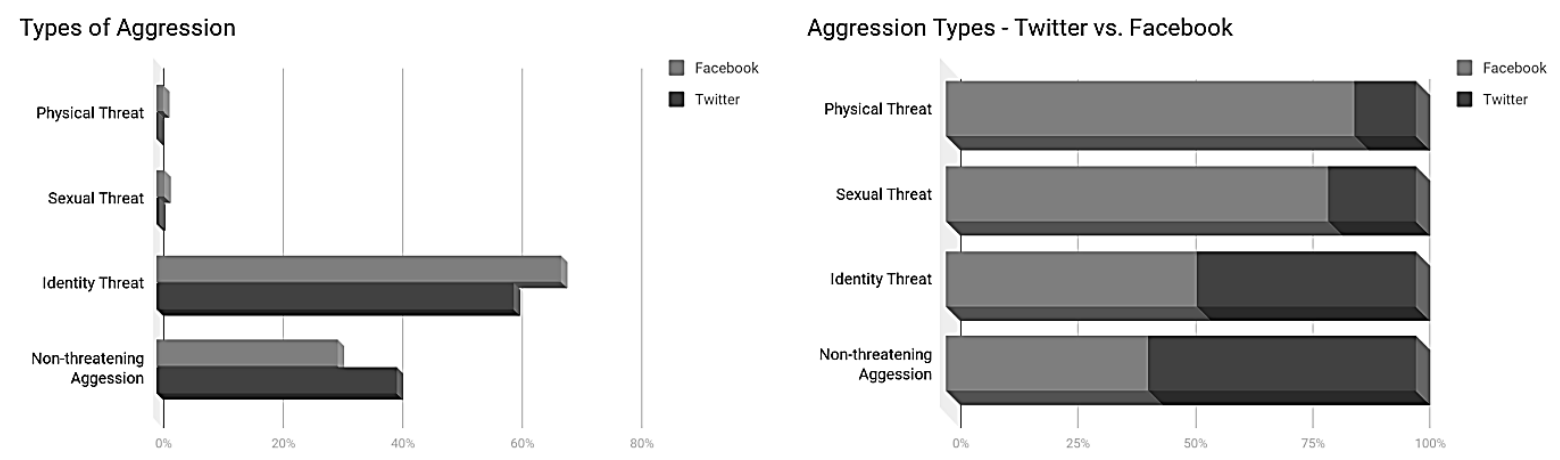

I further analysed the target of attack in cases of identity threat (Figure 5). The results indicated that people are overwhelmingly aggressive about political affiliation. Asides from this, people get aggressive about gender roles, religion and origin / country of residence. Caste and race are not very significant sources of aggression in this dataset. There could be a couple of reasons behind these results - one is, of course, the dataset itself which was largely collected from political domain. The other could also be the current discourse in the society - for example, in recent times, religion has become more significant than caste as the identity markers and so it has attracted more attack. If we look at the proportion of different kinds of attack on the two platforms, it throws some interesting but not very unexpected results. Twitter comes out to be significantly more gendered and also more aggressive about one's origin. On the other hand, Facebook is probably more concerned about the religion, race and caste of the individuals (despite the fact that race and caste, overall, do not trigger aggression in majority of cases) (Figure 5). These could again be explained by the demographic profile of the users and their concerns and needs to be further studied.

Figure 5: Different types of identity aggression and their proportion across the two platforms
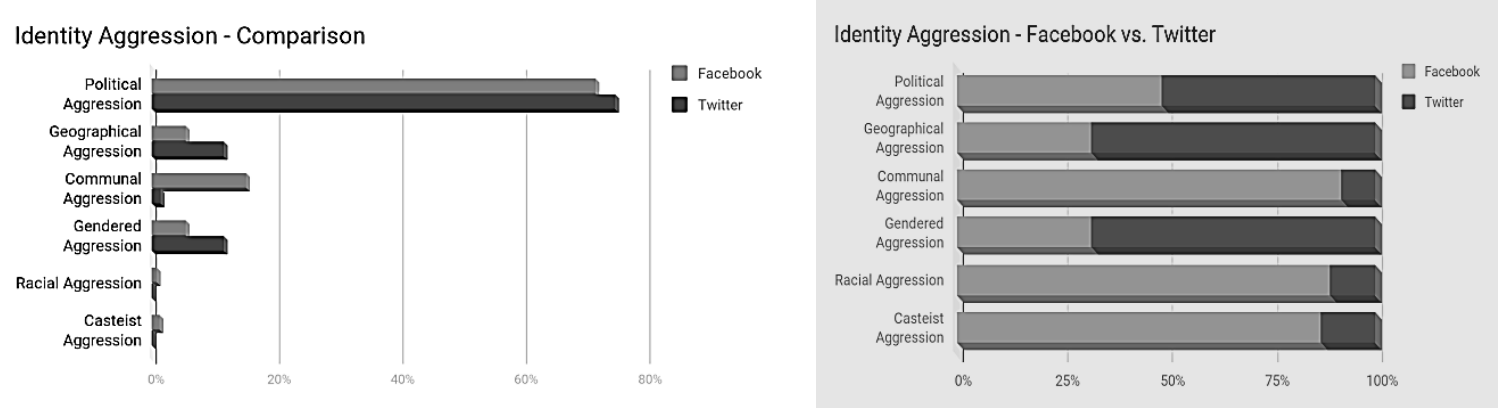


\section{Automatic Detection of Aggression}

Going by the study presented above, it is evident that aggression is quite prevalent on social media platforms like Facebook and Twitter. Even though all of the aggressive comments need not be and should not be reported or censored, some of those definitely needs to be automatically recognised and flagged. As such, we conducted some experiments for automatic classification of comments as OAG, CAG and NAG. We discuss some of these experiments and their results in the following sections.

\section{EXPERIMENT 1: N-GRAM FEATURES}

In the first set of experiments, we experimented with approximately 13,800 Facebook comments in Hindi and English each. The complete dataset was divided into 80:20 train:test ratio, resulting in approximately 11,000 training instances and 2,800 test instances for both the languages (Table 1).

As is evident, it is a slightly biased dataset - the proportion of NAG is quite less in Hindi and very high in English. It is not very surprising given the fact almost all the data is generated by the native speakers of Hindi and it is noted quite often that speakers switch to their native language when expressing opinion or even when abusing someone (Begum et al. 2016). Since this proportion represents the actual proportion in the dataset and is representative of the pragmatic facts of language usage on social media, we decided to keep the imbalance instead of balancing it out. We used Support Vector Machines (SVM) of scikit-learn for training the model. The parameters were tuned using Grid Search and 5-fold cross-validation. The performance of classifiers on test set with different combinations of features is given in Table 2 .

Table 1: Proportion of different classes in Facebook

\begin{tabular}{|l|c|c|c|c|}
\hline \multicolumn{1}{|c|}{ Class } & \multicolumn{2}{|c|}{ Hindi } & \multicolumn{2}{c|}{ English } \\
& Train Set & Test Set & Train Set & Test Set \\
Covertly Aggressive (CAG) & 4358 & 1107 & 2633 & 693 \\
Overtly Aggressive (OAG) & 4479 & 1138 & 1725 & 429 \\
Non-Aggressive (NAG) & 2213 & 518 & 6642 & 1678 \\
\hline
\end{tabular}

Table 2: Results of the n-gram experiments

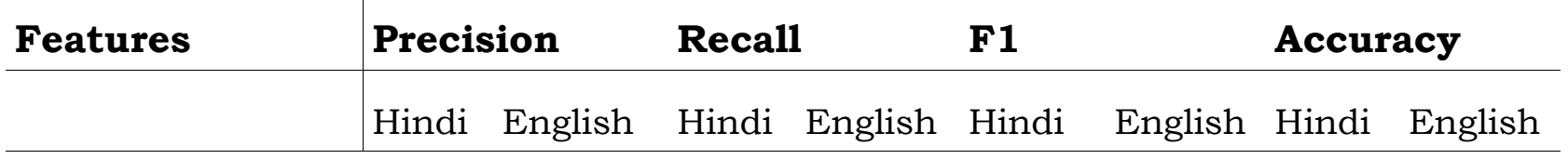

Character $\mathrm{n}$-gram features

\begin{tabular}{l|llllllll}
\hline $\mathrm{CB}+\mathrm{CT}(\mathrm{C} 1)$ & 0.61 & 0.59 & 0.59 & 0.64 & 0.59 & 0.57 & 59.03 & 63.57 \\
$\mathrm{CB}+\mathrm{CT}+\mathrm{CF}(\mathrm{C} 2)$ & 0.62 & 0.59 & 0.61 & 0.64 & 0.61 & 0.58 & 60.84 & 63.96 \\
$\mathrm{CB}+\mathrm{CT}+\mathrm{CF}+\mathrm{CFI}$ & 0.62 & 0.60 & 0.61 & 0.64 & $\mathbf{0 . 6 1}$ & $\mathbf{0 . 5 9}$ & 61.02 & 64.17
\end{tabular}


(C3)

\begin{tabular}{l|llllllll}
\multicolumn{1}{l}{ Word n-gram features } \\
\hline WU (W1) & 0.61 & 0.59 & 0.58 & 0.64 & 0.58 & 0.57 & 58.34 & 63.82 \\
WU+WB (W2) & 0.61 & 0.60 & 0.59 & 0.64 & $\mathbf{0 . 5 8}$ & 0.58 & 58.63 & 64.03 \\
WU+WB+WT (W3) & 0.60 & 0.61 & 0.59 & 0.65 & 0.58 & $\mathbf{0 . 6 1}$ & 58.60 & 64.82
\end{tabular}

Combination of character and word n-gram features

\begin{tabular}{l|llllllll}
\hline $\mathrm{C} 1+\mathrm{W} 1$ & 0.61 & 0.59 & 0.60 & 0.64 & 0.59 & 0.57 & 59.61 & 63.61 \\
$\mathrm{C} 1+\mathrm{W} 2$ & 0.62 & 0.59 & 0.60 & 0.64 & 0.60 & 0.57 & 60.00 & 63.71 \\
$\mathrm{C} 1+\mathrm{W} 3$ & 0.62 & 0.60 & 0.60 & 0.64 & 0.60 & 0.58 & 60.30 & 64.03 \\
$\mathrm{C} 2+\mathrm{W} 1$ & 0.62 & 0.59 & 0.61 & 0.64 & 0.61 & 0.58 & 60.95 & 63.93 \\
$\mathrm{C} 2+\mathrm{W} 2$ & 0.62 & 0.60 & 0.61 & 0.64 & 0.61 & 0.58 & 61.24 & 64.07 \\
$\mathrm{C} 2+\mathrm{W} 3$ & 0.63 & 0.60 & 0.61 & 0.64 & 0.61 & 0.58 & 61.35 & 64.18 \\
$\mathrm{C} 3+\mathrm{W} 1$ & 0.62 & 0.60 & 0.61 & 0.64 & 0.61 & 0.59 & 61.24 & 64.14 \\
$\mathrm{C} 3+\mathrm{W} 2$ & 0.63 & 0.60 & 0.61 & 0.64 & 0.61 & $\mathbf{0 . 5 9}$ & 61.49 & 64.21 \\
$\mathrm{C} 3+\mathrm{W} 3$ & 0.63 & 0.59 & 0.62 & 0.64 & $\mathbf{0 . 6 1}$ & 0.59 & 61.53 & 64.14
\end{tabular}

Both English and Hindi show a comparable performance, with Hindi performing slightly better than English. The best F1 score for Hindi was 0.61 which was produced with a combination of word uni- (WU), bi- (WB) and tri(WT) grams and character bi- $(\mathrm{CB})$, tri- $(\mathrm{CT})$, four- $(\mathrm{CF})$ and five- $(\mathrm{CFI})$ grams. On the other hand, the best performance of English was achieved with word uni-, bi- and tri- grams, which achieved an F1 score of 0.61. It seems that character-level features work marginally better for Hindi than word-level features and it is the other way round for English. This is not very surprising given the fact that Hindi is morphologically richer than English. Combining character and word-level features does not help much in case of Hindi and it, in fact, reduces the performance slightly in English. The best models were taken as the baseline for this task.

\section{EXPERIMENT 2: AdDitional FEATURES}

In the second round of experiments, we combined the English and Hindi data together and experimented with a slightly smaller dataset. This was done for 2 reasons - some of our experiments with the combined Hindi and English dataset yielded a similar result as with separate datasets. Since the comments on the social media platforms are written both in Hindi and English together, we decided to remove the language identification step (which might introduce additional errors in the pipeline) and train a combined model for Hindi and English (and that may also work for codemixed data). The slight reduction in the training data was also based on the observation that the larger number of samples was not really helpful in improving the performance and it would be quicker to run experiments with a smaller dataset. 
For this second set of experiments, we experimented with 2 different datasets - one for Twitter and other for Facebook. Approximately 8900 Facebook comments and 4787 tweets were used for training and approx. 500 comments / tweets were used for testing. The models were trained using SVM and the parameters were tuned using Grid Search and 5-fold cross-validation. We also added some more features for this set of experiments which included presence or absence of certain topics in the text (the topics were extracted from the dataset itself), mean sentiment score of the text calculated as mean of the scores of individual scores of words obtained from Hindi and English SentiWordNet, presence of any kind of emoticon (assuming that presence of any kind of emoticon is nonaggressive), the presence or absence of swear word or lexicons (based on a Swear Word Lexicon and a Slang Dictionary) and presence of non-standard punctuation and / or capitalization pattern in the text.

The use of these features led to a slightly better F1 score of 0.64 for both the tweets as well as facebook comments. The confusion matrix is given in Figure 6.

\section{ERROR ANALYSis}

As expected, the classifier does not show much confusion in between Overtly and Covertly Aggressive comments. On the other hand, covertly aggressive comments are not at all recognised properly and it seems to be equally divided across the 3 categories. This is expected, since by definition, it is rather difficult to recognise covert aggression using surface-level textual features. However, surprisingly, the classifier also seems to be quite confused in between the OAG and NAG, which was not expected. Let us take a look at a couple of NAG misclassified as OAG (a and b below) and viceversa (c and d).

(a) sonia is only bothered about her pay ... who cares for poor investors

(b) India reacted by killing muslims who allegedly eat beef. we also stopped cricket with Pak and banned movies that featured Pak actors. Revenge completed.

(c) RSS should be declared a hindu terrorist organisation.

(d) she is a female terrorist...

It seems that length of the comments play a significant role in the decision of the classifiers. Most of the shorter comments (less than $10-12$ words) are generally marked as NAG across the test set ((c) and (d) here). This could be attributed to the lack of sufficient features for making a proper decision. Another factor could be the fact that a large number of shorter comments were NAG (it includes comments involving general complements, thanking, etc.). As such there might be an implicit learning of length as a relevant feature for NAG. It need not be necessarily a bad thing as it shows that the classifier is not making decisions based on only a couple of words. However, this could be improved by giving higher weightage to the words in 
swear word lexicon, improving the lexicon to include more words as well as using a more robust measure (like TF-IDF) than frequency count for feature values. On the other hand, examples (a) and (b) do not seem to be an error on the part of the classifier and these were instances of disagreement among the human annotators as well.

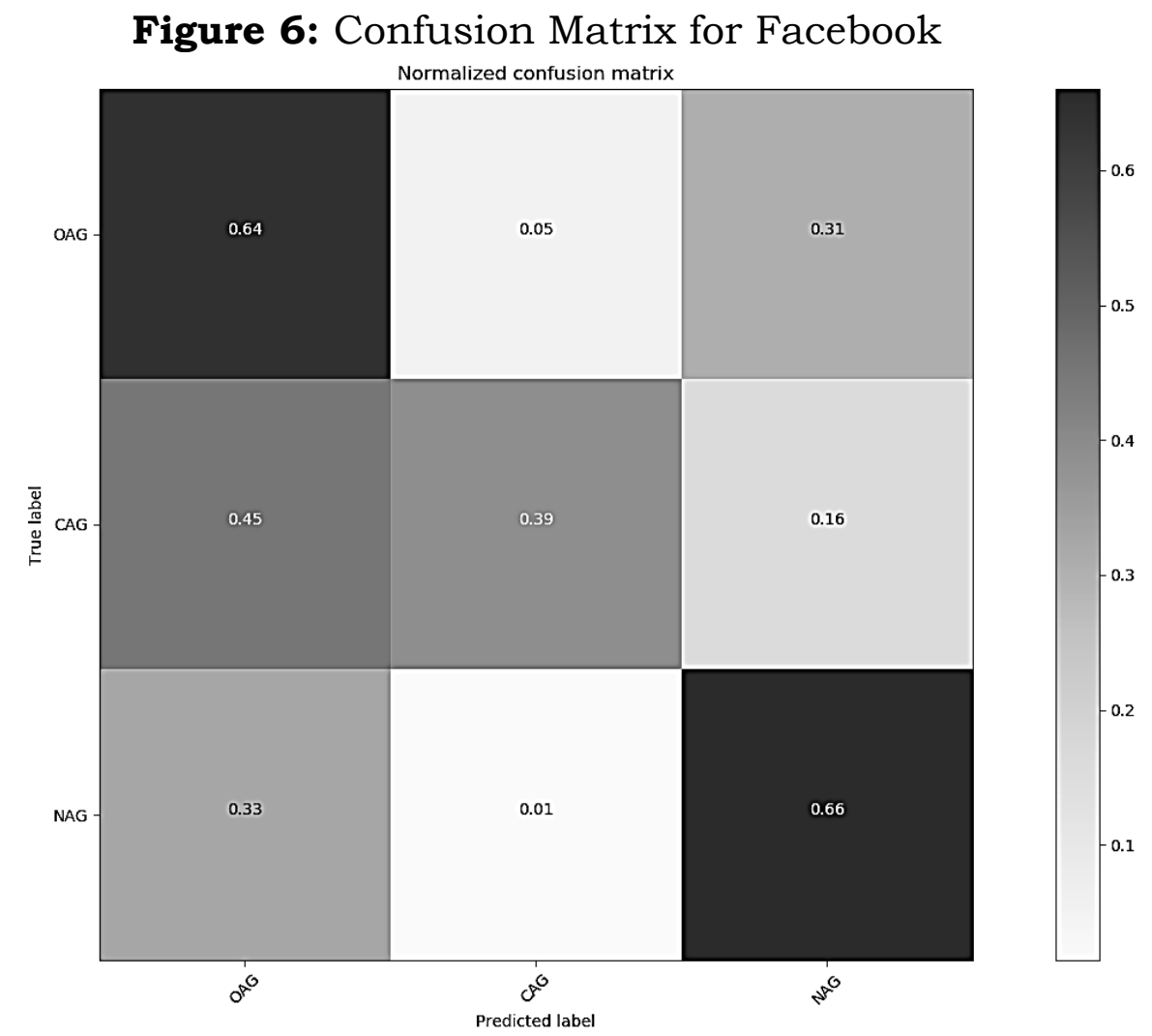

\section{ACCEPTABILITY OF THE CLASSIFIER: USER STUDY}

Aggression is a highly subjective phenomena and it is very difficult to identify it using only surface-level features. At the same time, there is a great amount of variability in the way aggression is interpreted among the speakers, which was also depicted in an overall low inter-annotator agreement of approximately $0.72^{\mathrm{vi}}$. Furthermore, a closer look at the errors showed that quite a lot of human judgments do not look reliable. Given this, F1 seems to be an inadequate way of accurately measuring system's performance since F1 scores are calculated only on the basis of comparison of the classifier's judgments against the judgment of only one human. As such, in order to appropriately judge the classifier's performance, the acceptability of the classifier's decisions among the users would be more appropriate measure than simple F1 or accuracy scores. Since in this case, classifier's decision is measured against that of a large number of human, we expect the statistics to gloss over the inherent subjectivity and bias in the judgments. Obviously this method cannot be used for acquiring better annotations because of several practical constraints. 
In order to do this, we designed a survey consisting of a total of 36 Facebook comments and the level of aggression as given by the classifier was presented to the user and they were asked to rate the appropriateness of the level on a scale of $1-5$, with 1 for the most inappropriate class and 5 for the most appropriate class. Out of these 36 comments, 18 were in English and 18 in Hindi. There were 6 comments in each category such that 2 of these comments were 'correctly' predicted by the classifier and 4 were 'wrongly' predicted. The survey was attempted by 31 respondents in English and 24 in Hindi. All the respondents were in the age group of $25-35$ and included a mix of linguists and non-linguists. The study was conducted more than 8 months after the manual annotation of the corpus and it was also attempted by 4 of the users who were involved in the initial annotation task.

A preliminary look at the response of the users gives a significantly different picture from what was given by the F1 scores. If we look at the judgment of the classifier for the four examples above, the prediction for example (a) is considered 'appropriate' (score of 4 or 5) by around $55 \%$ of respondents, neither bad nor good (score of 3) by another $22.5 \%$ while just around $22.5 \%$ considers it inappropriate (score of 1 or 2). Example (b) is considered appropriate by $77 \%$ of respondents, while only around $13 \%$ considers it inappropriate. Clearly the judgment made by the classifier seems to be right. On the other hand, for (c) almost $80 \%$ considered the classifier's judgment as inappropriate and almost $74 \%$ considers the judgment on (d) inappropriate and clearly shows that the classifier has made a mistake in these instances. Going by the majority votes, Figure 7 gives a summary of the number of comments in each category whose class assignment by the classifier was considered acceptable by the user.

Figure 7: Proportion of predictions by English and Hindi classifiers that were considered appropriate by users
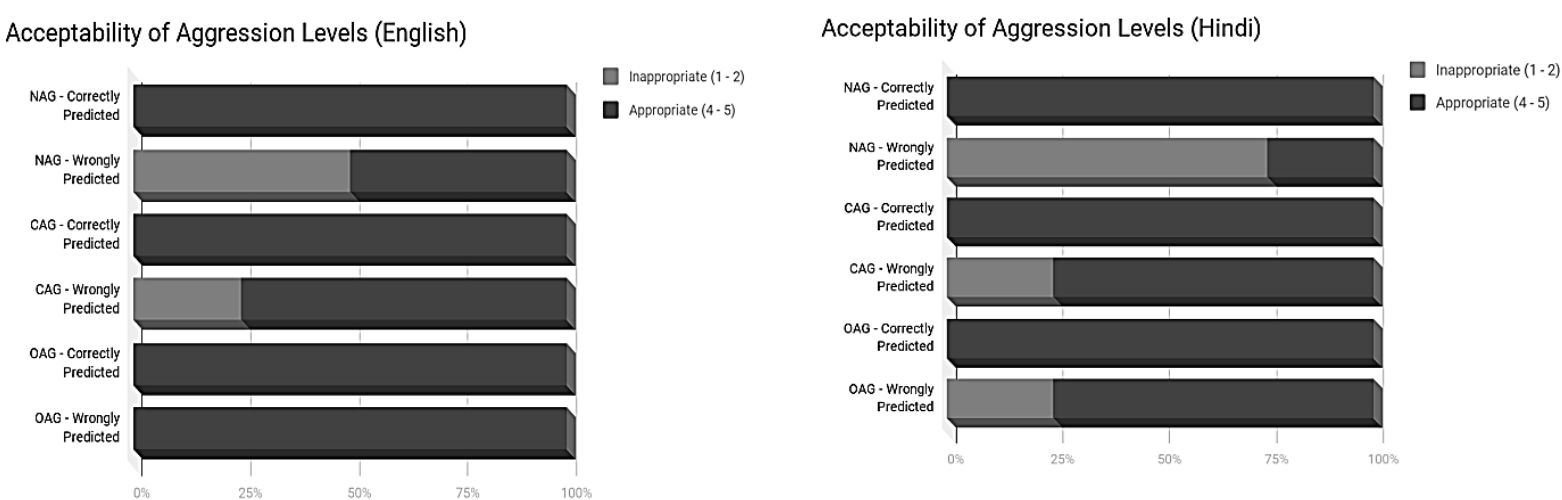

We see that in case of OAG in English, all the predictions made by the classifier is considered appropriate by a majority of users (including those which were judged to be wrong based on the previous annotation of the comment). In case of CAG in both English and Hindi and OAG in Hindi, only $25 \%$ of the 'wrong' predictions made by the classifier are considered inappropriate by users. In case of NAG, there is a larger agreement in 
between the annotators and the users. What is also noticeable is that none of the predictions that were considered correct were marked 'inappropriate' by the users - it is only in case of 'wrong' predictions that there is a disagreement and in most of the cases, decision given by the classifier is considered appropriate. Furthermore, a closer look at the 'inappropriate' classification reveals that most of the confusion is in between NAG and CAG, as expected. There are few instances of OAG being classified as NAG and those may be attributed to lack of sufficient features, as discussed above.

Going by these numbers, the acceptability of the prediction made by the classifier is much higher among a majority group of users than is depicted by the F1 score. It must be noted that the study was conducted at a relatively small scale (with limited number of participants as well as randomly sampled limited number of test instances) and the results might vary at a larger scale. Despite this, the study gives a good indication towards the classifier performing rather well with the users.

The study also goes on to show that it might be helpful to get the comments annotated by a larger group of annotators in order to reduce the effects of the inherent subjectivity of the task, which tends to get corrected by taking the majority opinion. However, it may not be always feasible to get a large amount of data annotated by a large number of annotators because of a variety of practical constraints.

\section{SUMMING UP AND THE WAY AHEAD}

In this paper, we have discussed the nature and extent of aggression on social media (more specifically Facebook), with special reference to Indian social media. We have looked at the relationship between abuse and aggression and also given an exposition of what makes people aggressive. We then go on to develop two classifiers for automatically detecting aggression on Facebook and Twitter in Hindi, English and Hindi-English code-mixed comments. Both the classifiers give a rather modest F1 score of 0.64. However, considering the fact that agreement among human annotators was 0.72 , the performance of the system seems to be quite acceptable and understandable. Moreover, a follow-up user study to test the acceptability of the predictions made by the classifier reveals a much greater acceptance for the classifier among the users than is illustrated by the F1 score.

It has been observed in the experiments that given the intrinsic subjectivity of the task itself, greater amount of data may not necessarily lead to a better performance of the classifier. One of the possible ways to improve the system further might be to improve the annotations themselves. This could be achieved by using a smaller dataset annotated with a large number of annotators, which will help in reducing the effects of the bias of one or two annotators. Moreover, as annotators become more experienced with the task, agreement among them increases. Thus using a group of 
experienced annotators might help in improving the annotations and the performance of the classifier.

\section{ACKNOWLEDGMENTS}

I would like to express my sincerest thanks to all my students and colleagues - Aishwarya, Tushar, Akshit, Ramprashanth, Monojit, Kalika, Atul, Mayank, Dhirendra and Deepak - who have contributed immensely to this research in different ways including help with the annotations and experiments. I would also like to thank Microsoft Research India for providing unrestricted research grant for the research.

\section{REFERENCES}

Begum, Rafiya, Kalika Bali, Monojit Choudhury, Koustav Rudra, and Niloy GANGULY. 2016. Functions of code-switching in tweets: An annotation framework and some initial experiments. In Proceedings of the Tenth International Conference on Language Resources and Evaluation (LREC 2016), Paris, France. European Language Resources Association (ELRA)

CAmbria, Erik, Praphul Chandra, Avinash Sharma, AND Amir Hussain. 2010. Do not feel the trolls. ISWC, Shanghai.

Chen, Ying, Yilu Zhou, Sencun Zhu, And Heng Xu. 2012. Detecting offensive language in social media to protect adolescent online safety. Privacy, Security, Risk and Trust (PASSAT), International Conference on Social Computing (SocialCom). Page 71-80

Cynthia Van Hee, Els Lefever, Ben Verhoeven, Julie Mennes, Bart Desmet, Guy De Pauw, Walter Daelemans, and VÉronique Hoste. 2015. Detection and fine-grained classification of cyberbullying events. In Proceedings of International Conference Recent Advances in Natural Language Processing (RANLP). Page 672-680.

CUlPEPER, JONATHAN. 2011. Impoliteness Using Language to cause Offense. Cambridge: Cambridge University Press.

DADVAR, MARAL, Dolf TrieschnigG, AND FRANCiSKA DE Jong. 2014. Experts and machines against bullies: a hybrid approach to detect cyberbullies. In Advances in Artificial Intelligence. Berlin: Springer. Page 275-281.

HARDAKer, Claire. 2010. Trolling in Asynchronous Computer-Mediated Communication: From User Discussions to Academic Definitions. Journal of Politeness Research. Language, Behaviour, Culture. Vol. 6(2). Page 215-242.

HARDAKER, ClAIRE. 2013. "Uh. . . . not to be nitpicky, ,,,, but...the past tense of drag is dragged, not drug." An overview of trolling strategies. Journal of Language Aggression and Conflict. Vol. 1(1). Page 58-86.

Karthik Dinakar, Birago Jones, Catherine Havasi, Henry Lieberman, AND ROSALIND PICARD. 2012. Common sense reasoning for detection, prevention, and mitigation of cyberbullying. ACM Transactions on Interactive Intelligent Systems (TiiS). Vol. 2(3). Page 18.

KROL, E. 1992. The whole internet: User's guide \& catalog. Sebastopol, CA: O'Reilly \& Associates, Inc. 
Kumar, Sudhakar, Francesca Spezzano, AND VS Subrahmanian. 2014. Accurately detecting trolls in slashdot zoo via decluttering. In Proceedings of IEEE/ACM International Conference on Advances in Social Networks Analysis and Mining (ASONAM). Page 188-195.

MoJICA, LuIS G. 2016. Modeling Trolling in Social Media Conversations. arXiv:1612.05310 [cs.CL]. https://arxiv.org/pdf/1612.05310.pdf [Retrieved on Feb 16, 2017]

Mihaylov, Todor, Georgi D Georgiev, AD Ontotext, and Preslav Nakov. 2015. Finding opinion manipulation trolls in news community forums. In Proceedings of the Nineteenth Conference on Computational Natural Language Learning, CoNLL. Page 310-314.

Nitin, AnkUsh Bansal, SidDhaRTha MahadeV Sharma, KaPil Kumar, AnUJ Aggarwal, Sheenu Goyal, Kanika Choudhary, Kunal Chawla, Kunal JAIN, MANAV BHASINAR. 2012. Classification of Flames in Computer Mediated Communications. arXiv:1202.0617 [cs.SI]. https://arxiv.org/pdf/1202.0617.pdf [Retrieved on Feb 16, 2017].

NitTa, Taisei, Fumito Masui, Michal Ptaszynski, Yasutomo Kimura, Rafal RZEPKA, AND KENJI ARAKI. 2013. Detecting cyberbullying entries on informal school websites based on category relevance maximization. In IJCNLP. Page 579-586.

Nobata, Chikashi, Joel Tetreault, Achint Thomas, Yashar Mehdad and Yi CHANG. 2016. Abusive Language Detection in Online User Content. In Proceedings of WWW2016. Page $145-153$.

SAX, SASHA. 2016. Flame Wars: Automatic Insult Detection. Stanford Reports. http://cs224d.stanford.edu/reports/Sax.pdf [Retrieved on Feb 20, 2017]

AnNa Schmidt AND Michael Wiegand. 2017. A Survey on Hate Speech Detection Using Natural Language Processing. In Proceedings of the Fifth International Workshop on Natural Language Processing for Social Media. Association for Computational Linguistics. Valencia, Spain. Pages $1-10$.

Schultz, JeFF. 2016. How Much Data is Created on the Internet Each Day? https://www.gwava.com/blog/internet-data-created-daily. Retrieved on February 28, 2017.

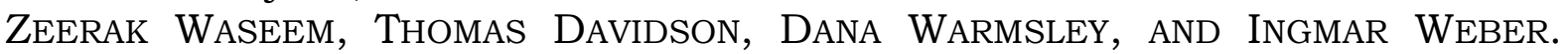
2017. Understanding abuse: A typology of abusive language detection subtasks. In Proceedings of the First Workshop on Abusive Language Online. Association for Computational Linguistics. Pages 78-84. 


\section{APPENDIX \\ The Aggression Tagset}

\section{Introduction}

This document gives detailed guidelines for annotating any text with information about aggression. The annotation is to be carried out using a hierarchical tagset of 3 tags, with 2 kinds of features - Discursive Role and Discursive Effect. This document gives a description of these categories and the features and how those could be employed during annotation.

\section{The Tagset / Annotation Scheme}

\begin{tabular}{|l|l|l|l|l|}
\hline Code & Aggression Level & TAG & \multicolumn{2}{|c|}{ Discursive Features } \\
\cline { 3 - 5 } & Discursive Role & Discursive Effect \\
\hline 1.1 & $\begin{array}{l}\text { Overtly } \\
\text { Aggressive }\end{array}$ & OAG & Yes & Yes \\
\hline 1.2 & $\begin{array}{l}\text { Covertly } \\
\text { Aggressive }\end{array}$ & CAG & Yes & Yes \\
\hline 1.3 & Non Aggressive & NAG & May be & No \\
\hline
\end{tabular}

2.1 Discursive Features: Discursive Role

\begin{tabular}{|l|l|l|}
\hline Code & Attribute & TAG \\
\hline 2.1 .1 & Attack & ATK \\
\hline 2.1 .2 & Defend & DFN \\
\hline 2.1 .3 & Abet & ABT \\
\hline
\end{tabular}

2.2 Discursive Features: Discursive Effect (Threats)

\begin{tabular}{|l|l|l|}
\hline Code & Attribute & TAG \\
\hline 2.2 .1 & Physical Threat & PTH \\
\hline 2.2 .2 & Sexual Aggression & SAG \\
\hline 2.2 .9 & $\begin{array}{l}\text { General Non-threatening } \\
\text { Aggression }\end{array}$ & NtAG \\
\hline 2.2 .4 & Identity Threat & ITH \\
\hline 2.2.5 & Curse / abuse & CuAG \\
\hline
\end{tabular}

\subsubsection{Discursive Features: Identity Threat}

\begin{tabular}{|l|l|l|}
\hline Code & Attribute & TAG \\
\hline 2.2.4.1 & Gendered Aggression & GAG \\
\hline 2.2.4.2 & Racial Aggression & RAG \\
\hline 2.2 .4 .3 & Communal Aggression & CoAG \\
\hline
\end{tabular}




\begin{tabular}{|l|l|l|} 
2.2.4.4 & Casteist Aggression & CaAG \\
\hline 2.2.4.5 & Political Aggression & PAG \\
\hline 2.2.4.6 & Geographical Aggression & GeAG \\
\hline
\end{tabular}

\section{Annotation Convention and Span}

On the top-level, the kind of aggression is annotated. At the second-level the relevant features - discursive activity and discursive effect - are annotated. Please note that discursive effect is annotated only for aggressive posts.

Annotation is carried out at the document level - it could be a complete post, a comment or any one unit of the discourse. In general, the top-level categories and discursive role will be annotated for the whole of the document. Discursive effects, however, can be marked for the whole of the document or a part of the document depending on what is happening in that particular post.

\section{Annotation Guidelines}

\section{Aggression Level}

At the top-most level, the post / comment / text is divided into three broad aggression levels as discussed below

\subsection{Overtly Aggressive (OAG)}

Any post / comment / text in which aggression is overtly expressed - either through the use of specific kind of lexical items or lexical features which is considered aggressive and / or certain syntactic structures is to be annotated using this label. It is a direct attack against the victim and involves use of commands, directives and specific kinds of lexical items An example is given below. vii

1. अबे कन्हइया सुना इंसहल्लाह (लाल चड्ढी) गैंग देल्ही $\mathrm{mcd}$ में 31 सीट पे चुनाव लड़ा ओर इन्हें कुल 51 वोटों से आजादी मिली है। कमाल हो गया बे।

Oye Kanhaiya, I have heard that Insaallah (red chaddi) gang contested election on 31 seats in Delhi MCD and they got azadi (freedom) by a total of 51 votes. It is amazing.

\subsection{Covertly Aggressive (CAG)}

Any post / comment / text in which aggression is not overtly expressed. It

- is indirect attack against the victim

- is often packaged as (insincere) polite expressions (through the use of conventionalised polite structures),

- includes sarcasm and satirical attacks. 
An example is given below

1. Harish Om kya anti-national ko bail mil sakti hai? ? (:) Harish $\mathrm{Om}$ can an anti-national get bail?

\subsection{Non-aggressive (NAG)}

This label should be given to all those human speech samples which do not exhibit aggression in any form. An example is as given below

1. Desh ka agla Jaiprakash..Agla Ambedkar yehi hai..

This is the next Jaiprakash and Ambedkar of the country.

\section{Discursive Features}

Two features - discursive role and discursive effect - will be annotated at Level 2. While both aggressive as well as non-aggressive posts will be annotated for the kind of discourse activity that they are performing, only aggressive posts will be marked for the discursive effect they are producing.

\subsection{Discursive Role}

It refers to the role of the current post / comment in the ongoing discourse and it could be one of three kinds, discussed below.

\subsubsection{Attack (ATK)}

Any comment / post which attacks a previous comment / post. It can only be aggressive. An example is given below:

1. Muh kala hai dogle ka dil bhi kala gaddar hai mujhe tum dikh jaye sala juta marunga dogala deshdrohi

This hypocrite has lost his face, his heart is also bad, as soon as I shall see you moron, I will hit you with a shoe, you hypocritical antinational.

\subsubsection{Defend (DFN)}

Any comment / post which defends or counter-attacks a previous comment / post. The previous comment / post must be an attack and the current one should be in support of the victim. It could be both aggressive as well as non-aggressive. An example of each case is given below:

1. Kitna dukhi hai bhai tu, lagta hai teri pool kholdi kanhaiya ne. Agar tu jo ilzam uspe laga raha hai wo sach hai toh wo kiyon jail me nahi hai. (Covertly aggressive)

How sad you are bro. It seems that Kanhaiya has shown your true face. If the accusation that you are labelling on him is true then why is he not in the prison. 
2. Av tak chargesheet file nhi kar payi h Delhi police...Aur lab ne is bat Ko confirm kiya ki anti-national slogan me Kanhaiya ki aawaz nhi h.ye dusre logo no kiya.kon kiya hoga ...Samjhte hi hoge. (Nonaggressive)

Delhi Police has not been able to file chargesheet till now. And the lab has confirmed that the anti-national slogan does not contain the voice of Kanhaiya. It has been done by other people. You must have an idea who has done it.

\subsubsection{Abet (ABT)}

Any comment / post which lends support and/or encourages a previous aggressive comment / post. The previous comment / post must be an attack and the current one should be in support of the aggressor. It could be both aggressive as well as non-aggressive.

1. Great sachchai likha aapne You have written great truth

Important Annotation Note: In cases of aggressive comments, it might be difficult to decide between attack and defend / abet. In such cases we annotate a comment as defend / abet ONLY when there is an explicit defense or abetment of a previous comment; otherwise we assume that it is an attack.

\subsection{Discursive Effects}

It refers to the kind of aggression in the current post / comment. This feature is available only for aggressive posts / comments.

\subsubsection{Physical Threat (PTH)}

Any aggressive post / comment that directly threatens to physically harm or hit the victim. It

- Threatens to hurt the victim (an individual or a community) physically or even kill her/him

- Includes suicide intentions, mass killings, etc.

- is potentially physically aggressive (verbal aggression transforming into physical aggression)

1. abey saaley haarami teri aukaat kya hai tu hug dega aaja bihar tewra wIT KAR RAHA HU ....MAREGA TU PAKKA .. JUB TAK DELHI ME HAI APNI KHUSIYA MANA MAI THOKUNGAA TERE KO

You moron, illegitimate son, what is your standing, you will pee in your pants. You come to Bihar, I am waiting for you. You will definitely die. You celebrate the time till you are in Delhi. I shall shoot you. 


\subsubsection{Sexual Threat / Aggression (SAG)}

Verbal aggression referring to the actual activity of sex or related stuff. It

- is graphic depiction of the actual act of sex.

- threaeans to actually carry out these acts (unratified expressions) against the victim.

1. Bhosri ke jab kuchh pata nahi hai to bolta kyu hai ja ke apni gaar marwa halala me.

You fucker, when you do not know anything then why do speak. Go and get yourself fucked in Halala.

\subsubsection{General Non-threatening Aggression (NtAG)}

Any act of aggression aimed towards the personal attributes like intelligence, physical features (but not really in relation to one's race or gender) or any other such attribute without giving out any kind of threat. It

- is aggression against individual traits and choices like color of the house, choice of food (non-communal), etc.

- includes most instances of personal insults, cyberbullying, etc.

An example is as given below.

1. Bhut kam jankari $h$ babu tmko.general caste ki aabadi $15 \%$ se $v$ kam h.general cat ka MATLAB hi h open category.

You know very little, boy. The population of general caste is less than $15 \%$. The meaning of general category is open category.

\subsubsection{Identity Threat (ITH)}

It threatens to one or more of the identities of the victim and includes aggression directed at social groups, communities, etc that the victim belongs to. The details of different classification of identity threat is given below.

\subsubsection{Curse / Abuse (CuAG)}

An act of aggression that involves cursing or abusing the victim without referring to any of the identifying attributes discussed above. An example is given below.

1. Mujahid Irfan meri kaonsi pool Di iss ghade kanhaiya ne Mujahid Irfan what has this ass Kanhaiya revealed about me?

2. भौं भौं भौं भौं भौं भौं

Sound of dog barking

\subsubsection{Gendered Aggression (GAG)}

Verbal aggression aimed towards the stereotypical gender roles of the victim as well as the aggressor. It also includes aggressive reference to one's 
sexuality and sexual orientation. It

- Attacks the victim because of / by referring to her/his gender (includes homophobic and transgender attacks)

- includes attack against the victim owing to not fufiling gender roles assigned to them or fulfiling the roles assigned to another gender

Some of the examples are given below.

1. tere ma se puch sale tera bap kon $h$

Go and ask your mother who your father is.

2. Napushank tha Nehru... lesbo thi indira

Nehru was impotent, Indira was a lesbian

3. Is hijray Rajnath ko chori pahna do

Give bangles to this eunuch Rajnath

\subsubsection{Racial Aggression (RAG)}

Verbal aggression aimed towards the skin color as well as ethnic identity / origin of the victim.

An example include

1. Ye chinkis ki bohat jal rahi he hamare desh ki Badti Milatry Power dekha kar. Sale china valo khud ke desh me original mal banana sikh lo pehle fir hume samjao.

These chinkis (Chinese) are so jealous of the increasing military power of our country. You moron Chinese first learn to manufacture the original stuffs then teach us.

\subsubsection{Communal Aggression (CoAG)}

Aggression against the real/presumed religious affiliation / identity / beliefs of the victim. An example from the corpus are given below.

1. Is nalayk ko koi ye bataye ki jaha bhi Muslim bahut jyada ho jate $\mathrm{h}$ waha hinduo ko kyo nahi jine dete Pakistan or bagladesh me hindu ka kya hal $h$ kashmiri pandit bisthapit kyo $h$ karana or godhara me hinduo par atyachar kisne kiya

Somebody tell this idiot that wherever there is a large number of Muslim, why are Hindus not allowed to survive there? What is condition of Hindus in Pakistan and Bangladesh? Why were Kashmiri Pandits forced to migrate? Who has victimised the Hindus in Karana and Godhara? 


\subsubsection{Casteist Aggression (CaAG)}

Verbal aggression towards the caste of the victim. An examples is given below.

1. Central govt $\mathrm{k}$ cabinet me dekho top $\mathrm{k} 10$ ya 20 ministers ko.1-2 ko chor yahi $15 \%$ wale $h$.

Look in the cabinet of central government. Among the top $10-20$ ministers, besides $1-2$, they are only these people with 15\% reservation.

\subsubsection{Political Aggression (PAG)}

Verbal aggression referring to one's political stand, beliefs and views. It

- is aggression directed against the victim for her/his presumed / actual affinity / membership to a particular political group / community

- is aggression against the political group / community itself

An example as following

1. ye Wahi insan hai na jo kahatha hai Bharat tere tukde honge inshallah inshallah iski maa ki ankh leftits log hai ye chutiye log ye saal Khatha hai Bharat ki Barbadi tak jung raheghi hamre desh kutte mar nahi skate hum....??? kutta ki dooom hai ye

This is the same person who says that India, you will be divided inshallah inshallah. This motherfucker is a leftist. Idiots, this moron say that there will be war till the destruction of India. Can our country not kill the dogs??? He is twisted like a dog's tail.

\subsubsection{Geographical Aggression (GeAG)}

Verbal aggression aimed towards the geographical region, area, country or state which one belongs to. It

- is aggression aimed at the victim referring to one's place of birth / origin / living

- 'Geographical' in this case could imply a small area like a locality to the whole of the Earth and everything in between which one's identity is attached with

An example is given below

1. Kahe ganda jhuth bolte ho sharminda h ki tum bihar se ho Why do you speak dirty lies. I am ashamed that you are from Bihar.

\section{Important Annotation Notes:}

- It may seem that certain comments may be annotated for more than one discursive effect. While annotating we should choose ALL the discursive effects that a comment shows.

- Most of the comments above (in the examples) contain one or the other form of 'abuse' - in those cases the comments must be marked 'abuse' and also at least one more effect must be marked in such 
cases. So any comment will have a minimum of two effects, if it contains abuse.

- If identity threat is exhibited by a comment then its sub-type(s) also need to be marked - please note that annotators are free to mark more than one kind of identity threats if it is present in the comment.

- If a comment is marked as exhibiting General Non-threatening Aggression then it cannot be marked for any other effect. In other words, any comment can be marked as General Non-threatening aggression only if it does NOT contain any other kind of threat/aggression. However, General Non-threatening aggression can also contain abuse and if it does, it should be marked so. Thus, NtAG comments can be only for abuse in addition to itself and nothing else.

\section{Notes}

i. 'Ratified' verbal behaviour refers to those behaviours which are expected and considered acceptable in a given context. For example, 'banter' could be a ratified aggressive behaviour among close friends. Similarly, aggression could be ratified on some online platforms. Since such behaviour is considered acceptable within given contexts, these cannot be considered criminal or even an undesirable behaviour. As such, it is necessary to make a fundamental distinction in between the ratified and unratified aggressive behaviour

ii. This comment is in the context of a widespread debate around the supposed role of student politics and student leaders in the country. The debate brought to the fore several biases of the common people including the assumption that the students engaged in political activities do not complete their studies on time in order to continue being a student and consequently waste the money and resources.

iii. The term 'discursive effects' could be understood as the effect / connotation that is produced by the aggression in the discourse - this allows us to subsume even those cases where the threat is not overt but could be deduced from the context

iv. The annotation guidelines is available on this link https://www.dropbox.com/s/pi5qtrhvmvywtky/aggression_tagset-v2glossed.pdf?dl=0

v. For example, in a task like part-of-speech annotation, where the number of tags is usually much larger than the current task, the agreement is usually above 0.95. Even for more subjective tasks like sentiment / opinion marking, the inter-annotator agreement is usually above 0.80

vi. The score is low only in comparison to some of the other more objective tasks however, for tasks as subjective as these, as I mentioned earlier, it is a reasonably good agreement score.

vii. All the examples given in this document are 'real-life' examples and they are reproduced exactly as they appear in the actual post / comment and form part of our corpus. 